
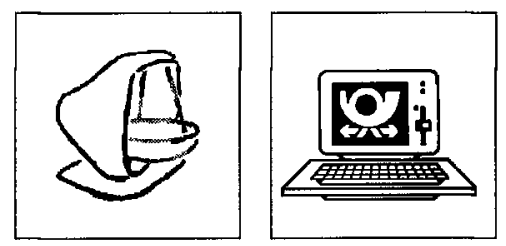

Das Internet-Programm des EDV-Gerichtstages

Wer sollte (und kann) was im Internet publizieren?

juris im Internet

Nene Software-Trends

Planungsfragen

Orientierungsfragen

\title{
Arbeitskreis "Internet"
}

\section{Maximilian Herberger}

Ein prägendes Thema des diesjährigen EDV-Gerichtstages ist das Internet. War noch vor einem Jahr nicht klar abzusehen, ob möglicherweise neue Anbieter dem Internet den Rang ablaufen würden, scheint diese Frage jetzt (auf mittlere Sicht) entschieden: Alle Anbieter von Online-Angeboten haben sich "um" das Internet gruppiert und bieten Übergänge dorthin an. Das Internet steht damit im Zentrum der Ereignisse. Planerische Bemühungen müssen dem Rechnung tragen. Der EDV-Gerichtstag hat bereits eine Adresse im Internet: http://edvgt.jura.uni-sb.de/edvgt/start.htm

Um allen Teilnehmern, soweit noch nötig, die unverzichtbaren Internet-Basisinformationen zu vermitteln, finden beginnend am ersten Tag um 12 Uhr stündlich Internet-"Grundkurse" im Computerraum der Juristen statt ("Internet kompakt"). Im Mittelpunkt steht dabei die Frage nach den Zugangsmöglichkeiten sowie der benötigten Software samt einigen ersten Bemerkungen zum Orientierungsproblem im Internet. Die Grundelemente des HTML(Hypertext Markup Language)-Standards werden vorgestellt. Frei verteilbare Software, die dabei erwähnt wird, kann sogleich mitgenommen werden. Der Internet-Arbeitskreis am zweiten Tag, von dem nun die Rede sein soll, kann auf diese Art und Weise mit einem einheitlichen Mindestkenntnisstand rechnen.

Die Fragen, die dann noch zu beantworten bleiben und im Arbeitskreis diskutiert werden sollen, betreffen - als offene Vorschlagsliste gedacht - die folgenden Themen.

Sinnvollerweise sollte sich das Internet-Programm des EDV-Gerichtstages nicht auf das Tagungsprogramm beschränken, sondern über das Jahr hinweg weitere Informationsangebote bereithalten. Dies setzt, soll nicht das Zufallsprinzip herrschen, eine Einigung auf Mindestrubriken und Zulieferverpflichtungen voraus.

Angesichts der Leichtigkeit, mit der im Internet elektronisch publiziert werden kann, müssen sich alle juristischen Institutionen die Frage stellen, ob sie weiterhin abseits stehen sollten. Im internationalen Vergleich fehlen in Deutschland noch Angebote von Institutionen, die anderswo in der Welt bereits rege im Internet aktiv sind. Dies gilt etwa für Parlamente, Ministerien und Gerichte. Im Arbeitskreis wollen wir die internationale Situation darstellen, um zu vergleichendem Fragen anzuregen.

Es steht zu erwarten, daß juris in zeitlicher Nähe zum EDV-Gerichtstag seinen Start ins Internet bekanntgeben wird. Unser Arbeitskreis bietet Gelegenheit, Wünsche und Erwartungen zu formulieren sowie Vorschläge zu unterbreiten.

Während Kenntnisse über den HTML-Standard sich gerade zu festigen beginnen, kündigen sich bereits neue Software-Trends an. Soweit man das abschätzen kann, wird die interaktive Internet-Anwendungen unterstützende Programmiersprache "Hot Java" maßgeblichen Einfluß gewinnen. Parallel dazu entstehen Script-Sprachen, die - teils an "Visual Basic for Applications" angelehnt - eine Integration der Internet-Angebote mit der Datenwelt des eigenen PC erlauben, und zwar in beiden Richtungen. Auch auf diese Werkzeuge wird der Arbeitskreis einen Blick werfen.

Der Erneuerungszyklus der Internet-Arbeitsumgebung hat ein Tempo angenommen, dem mit klassischen Planungsstrategien nicht mehr beizukommen ist. Es wird deswegen zu diskutieren sein, ob es überhaupt Möglichkeiten gibt, diese Entwicklung kompetent zu begleiten. Glücklicherweise bietet das Internet selbst Instrumente, die es erlauben, eine gewisse Planungskompetenz aufzubauen. Ob das dabei zu erreichende Niveau ausreichend ist, bleibt zu diskutieren.

Das - auch was juristische Fachinformation angeht - förmlich explodierende Informationsangebot im Internet wirft Orientierungsprobleme besonderer Art auf. Wer zeitökonomisch mit dieser Situation umgehen will, benötigt eine Strategie. Bausteine dazu will der Arbeitskreis gleichfalls liefern. 